\title{
A PARTICLE-CORE STUDY OF HALO FORMATION FOR A MISMATCHED BEAM IN A PERIODIC-FOCUSING SYSTEM ${ }^{*}$
}

\author{
Tai-Sen F. Wang ${ }^{* *}$ and Thomas P. Wangler, LANL, Los Alamos, NM
}

\section{Abstract}

This work is an attempt to explore possible techniques to graphically display the dynamics of halo particles for a mismatched beam in a periodic-focusing lattice. It is found that by using the particle-core model and a suitable change of the phase-space variables, stroboscopic plots similar to those made for the uniform-focusing channel can be created by strobing at the frequency of the core oscillation. This method does not require any smooth approximation to reduce the flutter due to the periodic focusing, and it is not limited by the constraint that the core-oscillation frequency has to be a subharmonic of the focusing frequency. The discussion will cover the applications to both the axisymmetric and the quadrupole-focusing channels. It is also found that, similar to the continuous-focusing system, the motion of halo particles, although focused by the externally applied periodic field, is strongly influenced by a parametric resonance.

\section{INTRODUCTION}

The particle-core model has successfully explained the dynamics of particles in the beam halo of a mismatched beam propagating through a uniformfocusing channel.[1-12] The simplicity of the model makes it possible to mathematically analyze the motion of a particle in the beam halo and provides a graphic picture using the stroboscopic plot made on the phase plane of a test particle. However, when attempting to apply the same model and method to a beam propagating through a periodicfocusing channel, one encounters difficulties both in mathematical analyses and in making the stroboscopic plots. The main cause of these difficulties is the flutter in the beam envelope and the particle orbit introduced by the varying focusing field.

In this paper, we will show that by using appropriate phase-space variables and by strobing at the frequency of the core oscillation, the flutter due to the periodic focusing can be greatly reduced. This method works best when the beam current is not very high, but it is also applicable to a wide range of parameter values. We will first introduce the new phase-space variables; then we will present the method and discuss the application of this new approach to

*Work supported by Los Alamos National Laboratory, under the auspices of the US Department of Energy.

** Email: TWANG@LANL.GOV a quadrupole-focussing system. Numerical examples will be given for a core with a Kapchinskij-Vladmirskij (KV) distribution of beam particles. The emphasis in this paper is to introduce the method. More studies using this approach will be reported in other publications.

\section{THE PARTICLE-CORE MODEL}

We consider a theoretical model which has a test particle and a continuous beam (the core) propagating in a periodic-focusing channel. We consider a core with a KV distribution of particles. The linear transverse focusing force is assumed to vary in the axial direction (the $z$-direction) according to $G F(k z)$ where $G$ is the maximal gradient of the focusing (or defocusing) strength, $F(k z)$ is a periodic function of $z$, and $k$ is the wave-number of the periodic-focusing system. The maximum of $|F(k z)|$ is assumed to be one.

We consider the axisymmetric focusing case first. Using the variable $\tau=k z$, the equations of motion for the beam envelope and the test particle are:

$$
\frac{d^{2} X}{d \tau^{2}}+Q^{2} X F(\tau)-\frac{\eta}{X}-\frac{1}{X^{3}}=0
$$

and

$$
\frac{d^{2} x}{d \tau^{2}}-\frac{L^{2}}{x^{3}}+Q^{2} x F(\tau)= \begin{cases}\eta x / X^{2} & , \text { for } x \leq X \\ \eta / x, & \text { for } x>X\end{cases}
$$

respectively, where $x=x_{r} \sqrt{k / \epsilon}, X=X_{r} \sqrt{k / \epsilon}, x_{r}$ is the transverse displacement of the particle from the symmetry-axis of the beam, $X_{r}$ is the beam envelope, $Q^{2}=q G /\left(m_{0} \gamma v^{2} k^{2}\right), \eta=q I /\left(2 \pi \epsilon_{0} m_{0} \gamma^{3} v^{3} k \epsilon\right)$, and $L=L_{r} /\left(m_{0} \gamma v \epsilon\right) ; q, m_{0}$, and $L_{r}$ are the charge, the rest mass, and the angular momentum of the test particle, respectively; $\gamma$ is the relativistic mass factor, $v$ is the axial speed of beam particles, $I$ is the beam current, $\epsilon_{0}$ is the permittivity of free space, and $\epsilon$ is the unnormalized total beam emittance.

We now introduce a set of new variables defined by

$$
\begin{aligned}
& \qquad \begin{array}{c}
u=x / X \\
w=X^{2}(d u / d \tau)=X(d x / d \tau)-x(d X / d \tau), \\
u_{e}=X_{m} / X
\end{array} \\
& \text { and } \begin{array}{c}
w_{e}=X^{2}\left(d u_{e} / d \tau\right)=X\left(d X_{m} / d \tau\right)-X_{m}(d X / d \tau),
\end{array}
\end{aligned}
$$$$
\text { and }
$$

where the subscript $m$ is for a matched core. Rewriting Eqs. (1) and (2) using these new variables, we find that these equations then depend explicitly on $X$ instead 
of $F(k z)$. Results from both numerical experiments (see Fig. 1 below) and perturbation calculations show that the flutter in $u$ and $w$ is smaller than that in $x$ and $d x / d \tau$. One can also prove that the change of variables from $(x, d x / d \tau)$ to $(u, w)$ is a canonical transformation, and for particles inside the phasespace ellipse of the beam core, the new Hamiltonian is a constant of motion with respect to the time $s$ (defined by $d s=d \tau / X^{2}$ ). For particles outside the ellipse, the Hamiltonian is time-dependent and is nonintegrable.

The proposed approach here is to study the particle dynamics in the phase space of $(u, w)$ and to make the stroboscopic plot by taking snapshots of the particle's phase space at a certain fixed value, say, the maxima or the minima, of $u_{e}$. Numerical results have shown that the oscillation frequency of $u_{e}$ is not constant in the periodic-focusing case, and strobing at constant period creates larger spread of points in the stroboscopic plots. Note that in Eqs. (3) and (4), one can also choose to normalize to $X_{m}$ in place of $X$ to minimize the flutter in $u$ and $w$.

In quadrupole-focussing channels, the equations of the beam envelope and particle motion in the $x$ direction are

$$
\frac{d^{2} X}{d \tau^{2}}+Q^{2} X F(\tau)-\frac{2 \hat{\eta}}{X+Y}-\frac{\epsilon_{x}^{2}}{X^{3}}=0,
$$

and

$$
\frac{d^{2} x}{d \tau^{2}}+Q^{2} x F(\tau)=\frac{2 \hat{\eta} x}{\Xi_{x}\left(\Xi_{x}+\Xi_{y}\right)},
$$

respectively, where $X=X_{r} \sqrt{k}, Y=Y_{r} \sqrt{k}, X_{r}$ and $Y_{r}$ are the beam envelopes in the $x$ - and $y$-directions, respectively, $x=x_{r} \sqrt{k}, x_{r}$ is the displacement of the beam particle in the $x$-direction from the beam axis, $\hat{\eta}=q I /\left(2 \pi \epsilon_{0} m_{0} \gamma^{3} v^{3} k\right), \epsilon_{x}$ is the beam emittance in the $x$-direction, $\Xi_{x}=\sqrt{X^{2}+\xi}, \Xi_{y}=\sqrt{Y^{2}+\xi}, \xi=0$ when the particle is inside the beam, and $\xi$ is given by the solution of the equation $\left(x / \Xi_{x}\right)^{2}+\left(y / \Xi_{y}\right)^{2}=1$, when the particle is exterior to the beam, $y=y_{r} \sqrt{k}$, and $y_{r}$ is the excursion of the beam particle in the $y$ direction from the beam axis. The equations for the beam envelope and particle motion in the $y$-direction are similar. To generalize our method developed for axisymmetric systems to a quadrupole-focussing system, one can use the variables defined according to $u_{x}=x / X, w_{x}=X(d x / d \tau)-x(d X / d \tau), u_{e x}=$ $X_{m} / X, w_{e x}=X\left(d X_{m} / d \tau\right)-X_{m}(d X / d \tau)$, and similar definitions for the $y$-direction variables. Since both the beam envelope and the test particle now have two degrees of freedom, only the stroboscopic plots made for some special cases can be deciphered easily. Thus, we have to consider the $x$ - and $y$-motion of the particle separately by setting one of the coordinates to zero, e.g. $y=0$ and $d y / d \tau=0$. Besides, in addition to the breathing mode, the beam envelope can now also oscillate in a quadrupole mode. Since the frequencies of these two modes are very close, even when the test particle is limited to have only one degree of freedom, it still may resonate with either one or a combination of these two envelope modes.

\section{NUMERICAL EXAMPLES}

Figure 1 presents an example of a periodic-focusing channel showing that the flutter is less in $u$ than in $x$. The case studied is an axisymmetric focusing channel with $F(\tau)=\cos \tau$, and $Q^{2}=0.31966$. At zero beam current, the betatron phase advance of a beam particle is $90^{\circ}$ per period. At full beam current, $\eta=0.206$ corresponding to a depressed phase advance of $60^{\circ}$ per period for particles inside of the matched core. The initial values are: $u=1.23491, X=3.34883$, $u_{e}=1 / 0.9, w=d X / d \tau=0$, and $L_{r}=0$.

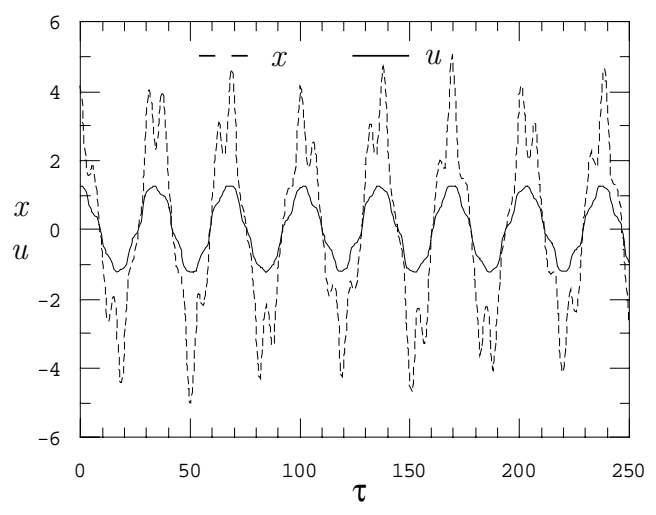

Fig. 1. The orbit $x$ and the quantity $u$ of a particle in a mismatched beam. The parameter values are described in the text.

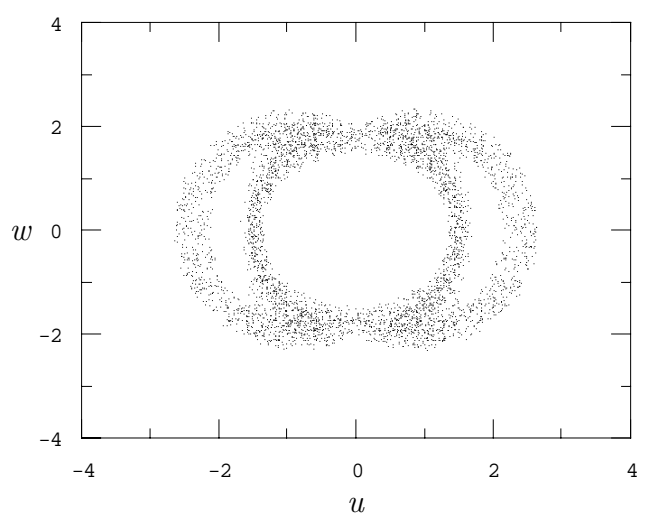

Fig. 2. The stroboscopic plot of the particle's position on the phase plane of $u$ and $w$, where the same parameter values considered in Fig. 1 were used in the computation.

Figure 2 shows a typical stroboscopic plot for one particle constructed by strobing at every local minimum of $u_{e}$, where the same focusing channel and beam conditions in Fig. 1 are considered. The initial position of the test particle is $(u, w)=(1.28776,0)$ in 
the phase space. In the figure, we see points scattered near an invariant curve in the Poincaré plot for the smoothed uniform-focusing channel. Figure 2 suggests that in this particular case, the averaged particle motion is in resonance with the core oscillation, and the orbit of the particle could be quasi-periodic or almost-periodic. The scattering of points is a general feature for particles outside the phase-space ellipse of the beam core. This is because a stroboscopic plot made for a particle in the periodic-focusing system is actually the projection of a higher dimensional "plot" onto the two-dimensional plane.[13] As the particle approaches toward to the beam ellipse, the dispersion of the strobed points decreases. For a particle inside the phase-space ellipse of the core, the strobed points fall exactly on an invariant circle as expected.

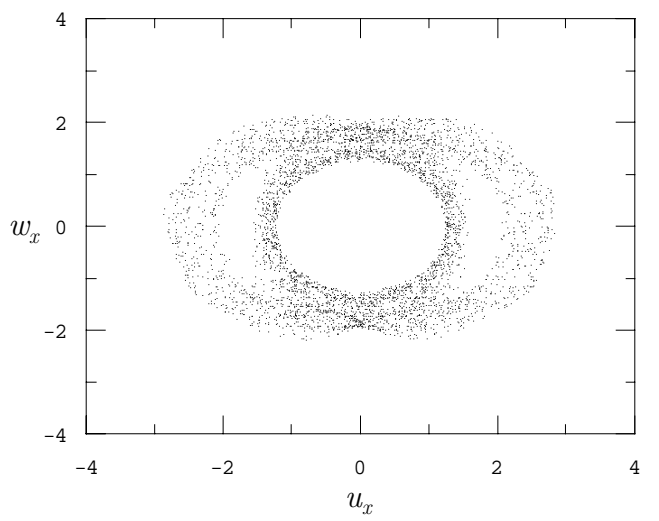

Fig. 3. Stroboscopic plot showing the resonance of a halo particle with the breathing mode oscillation of the beam envelope in a quadrupole-focusing channel.

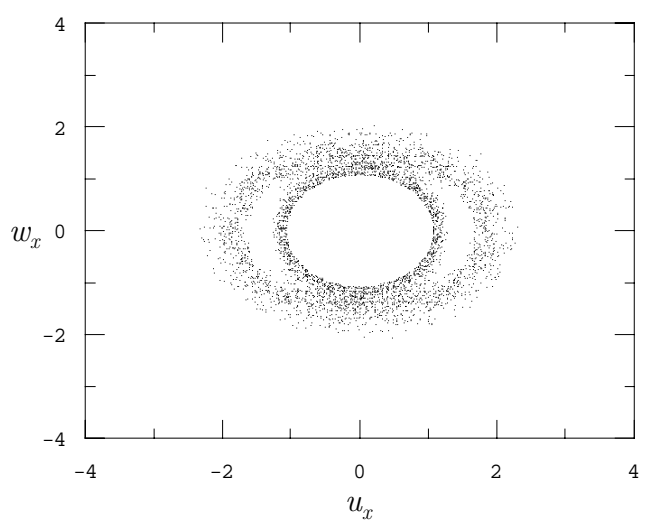

Fig. 4. Stroboscopic plot showing the resonance of a halo particle with the quadrupole mode oscillation of the beam envelope in a quadrupole-focusing channel.

Examples of stroboscopic plots for a quadrupolefocusing channel are shown in Figs. 3 and 4 for a particle in resonance with the breathing mode and the quadrupole mode of the envelope oscillation, respectively. The focusing and the beam parameters considered in these examples are: $F(\tau)=\cos (\tau)$, $\epsilon_{x}=\epsilon_{y}=\epsilon=1, Q^{2}=3.198$, and $\hat{\eta} / \epsilon=0.2502$. These parameter values correspond to a tune depression from $90^{\circ}$ to $70^{\circ}$ for a particles inside the matched beam. The initial conditions used are: $u_{e x}=u_{e y}=0.9$, $u_{x} \approx 1.26004$, for Fig. 3, and $u_{e x}=0.9, u_{e y}=1.1$, $u_{x} \approx 1.08235$, for Fig. $4 ; w_{e x}=w_{e y}=w_{x}=u_{y}=$ $w_{y}=0$ for both figures.

\section{CONCLUSIONS}

A new method has been developed to use the particle-core model for studying the dynamics of halo particles in a mismatched continuous-beam propagating through a periodic-focusing channel. It was shown that by applying appropriate transformations of phase-space variables and by strobing at the frequency of the core oscillation, one is able to create stroboscopic plots similar to the Poincaré plots made for particles in a uniform-focusing channel. This method is applicable to a wide range of parameter values without using any smooth approximation, and it is not limited by the constraint that the frequency of core oscillation must be commensurable with that of the focusing lattice. Numerical examples were given to illustrate the method by considering a beam with a KV distribution and an axisymmetric cosine transversefocusing force. We have discussed the possibility of extending this method to some limited cases in periodic quadrupole-focusing systems. It was also shown that, in spite of the complications brought in by the non-linear oscillations of the system, the motion of a halo particle is still strongly influenced by the resonance between motions of the particle and the core.

\section{REFERENCES}

[1] R. Jameson, Los Alamos Report LA-UR-93-1209 (1993).

[2] J. S. O'Connell, T. P. Wangler, R. S. Mills, and K. R. Crandall, Proc. of 1993 Particle Accelerator Conf., p. 3657 (1993).

[3] T. P. Wangler, Los Alamos Report LA-UR-94-1135 (1994).

[4] J. Lagniel, Nucl. Instrum. Meth., A345, 46 (1994); A345, 405 (1994).

[5] R. Gluckstern, Phys. Rev. Lett., 73, 1247 (1994).

[6] C. Chen, and R. C. Davidson, Phys. Rev. Lett., 72, 2105 (1994); Phys. Rev. E, 49, 5679 (1994).

[7] S. Y. Lee, and A. Riabko, Phys. Rev. E, 51, 1609 (1995).

[8] C. Chen, and R. A. Jameson, Phys. Rev. E, 52, 3074 (1995).

[9] T. P. Wangler, R. W. Garnett, E. R. Gray, R. D. Ryne, and T.-S. Wang, Proc. of XVIII Intl. Linear Accelerator Conf., 1996, CERN report CERN 96-07, p. 372.

[10] H. Okamoto, and M. Ikegami, Phys. Rev. E, 55, 4694 (1997).

[11] M. Ikegami, Phys. Rev. E, 59, 2330 (1999). M. Ikegami, and M. Mitzumoto, Proc. of XIX Intl. Linear Accelerator Conf., Chicago, 1998, (in press).

[12] T. P. Wangler, K. R. Crandall, R. Ryan, and T.-S. Wang, Phys. Rev. ST-AB, Vol. 1, No. 8, (1998).

[13] F. C. Moon and W. T. Holms, Phys. Lett., 111A, 157 (1985). 